(n)

\title{
Entretien avec Tim MURRAY
}

\begin{abstract}
BioTribune: Quels sont les sites Web que vous visitez dans le cadre de votre activité professionnelle?

Tim MURRAY : www.biospace.com. J'ai aussi souscrit à leur NewsLetter. www.biofind.com pour m'informer des rumeurs issues de l'industrie.

www.apbiotech.com est un bon site pour ce qui concerne le séquençage d'ADN. www.qiagen.com parce qu'ils sont leaders dans les produits d'extraction DNA/RNA et pour la qualité des informations techniques. Et enfin, www.marketing.org qui est un site interprofessionnel dont la mission est de regrouper les informations liées au marché du biomédical.
\end{abstract}

\section{BT : Quelle est votre approche du Web dans la relation client et quel est votre objectif?}

T.M. : Visible Genetics a ouvert deux sites Internet : un site lié a l'entreprise, visgen.com qui a vu le jour en 1999 et dont une nouvelle version va sortir prochainement, et un site dédié à notre gamme TRUGENE ${ }^{\mathrm{M}}$. www.trugene.com propose des fiches produit et des ressources techniques disponibles. Le site d'entreprise offre quant à lui, une approche plus générale de l'activité de la société à quelqu'un qui découvre Visible Genetics. Il est aussi une source de renseignements pour nos porteurs d'actions et investisseurs.

L'objectif est de mettre à la disposition de nos clients et des personnes intéressées, des sites complets et conviviaux où ils trouveront les toutes dernières informations relatives à notre activité.

\section{BT : Quelle est votre stratégie} concernant le contenu éditorial et les informations scientifiques ou techniques que vous diffusez sur internet?

T.M. : Le site Trugene.com a été conçu pour répondre aux attentes de quatre groupes d'audience : les cliniciens, le personnel de laboratoire, les commissions de remboursement et les patients. Le contenu de chaque section étant adapté au groupe d'audience. De plus, une fois par trimestre, nous éditons notre lettre d'information, VGInsight, que nous envoyons soit par e-mail soit par courrier. Les utilisateurs peuvent s'inscrire sur le site pour recevoir directement les futurs numéros de VGInsight. Le site Trugene.com permet aussi de diffuser de la vidéo et des présentations PowerPoint grâce a la technologie REAL. Un onglet est entièrement dédié au Multimédia.

BT : Quels outils ou services offrezvous ? Prévoyez-vous la possibilité de commander en ligne?

T.M. : Pour des produits réglementés comme les nôtres, il est difficile d'offrir à nos clients la possibilité de passer directement leurs commandes par l'intermédiaire du site. Aussi les commandes sont-elles enregistrées de façon traditionnelle. Par contre, nous offrons à nos clients la possibilité de télécharger différents modules de formation. Le site offre par l'intermédiaire du site MedScape CME Circle, un programme sur la résistance du VIH-1 (HIV-1 Resistance Monograph) agréé pour la FMC.

BT : Comment communiquez-vous sur le web pour faire connaître vos sites? T.M. : Visible Genetics s'est associé à d'autres sites médicaux comme MedScape qui sont capables de quantifier les visites effectuées par mois (mesure du nombre de fois où notre bannière a été appelée). MedScape enregistre les informations démographiques des cliniciens au moment de leurs inscriptions, nous connaissons indirectement le profil des personnes qui nous visitent. Le site Trugene offre aussi une longue liste de liens avec d'autres sites scientifiques ou professionnels. Nous sponsorisons des sites comme MedScape et HIVandHepatitis.com. Et en France nous travaillons plus particulièrement avec le site BIOTRIBUNE.COM qui accueille

\author{
Tim Murray \\ Product Manager, \\ Visible genetics Corp.
}

quelques pages d'informations en français sur notre gamme Trugene.

BT: Quels sont les outils de programmation que vous utilisez? Faites vous appel à des ressources externes? (from Dorin Grunberg, Web Master)

T.M. : Seuls quelques concepts graphiques sont réalisés à l'extérieur. Tout le reste est fait par Visible Genetics. Le site utilise les technologies Apache, Perl,PHP, PostgreSQL, et SSI. L'ensemble fonctionne sous le système d'exploitation OpenBSD 2.9 (UNIX). Je réalise quelques travaux graphiques avec Photoshop que je code avec HomeSite 4.5.

\section{BT : Prévoyez-vous de décliner vos} sites dans d'autres langues?

T.M. : Nous nous y intéresserons lorsque notre développement aura atteint un niveau tel que nous aurons les ressources appropriées pour créer des sites par langage ou par pays. Mais pour le moment, nous pensons que notre site en anglais répond aux besoins de tous nos clients, quel que soit leur pays d'origine.

BT: Quelle est votre vision de l'outil Internet dans le futur?

T.M. : Internet a fondamentalement changé notre façon de travailler. Dans le secteur médical, Internet sert de moyen d'information pour les patients et les médecins. Ce savoir étant facilement accessible, un patient peut disposer d'autant d'informations que son médecin. Pour les laboratoires et les fournisseurs, Internet est le moyen principal de communication et de collaboration. Commandes en direct, gestion des clients et autres fonctions permettront de rendre nos vies professionnelles plus faciles et plus efficaces. 